\title{
An Improved In Vivo Methodology to Visualise Tumour Induced Changes in Vasculature Using the Chick Chorionic Allantoic Membrane Assay
}

\author{
NASIDE MANGIR ${ }^{1,2}$, AHTASHAM RAZA ${ }^{1}$, JOHN W. HAYCOCK $^{1}$, \\ CHRISTOPHER CHAPPLE ${ }^{2}$ and SHEILA MACNEIL ${ }^{1}$ \\ ${ }^{1}$ Department of Materials Science Engineering, Kroto Research Institute, University of Sheffield, Sheffield, U.K.; \\ ${ }^{2}$ Royal Hallamshire Hospital, Urology Clinic, Sheffield, U.K.
}

\begin{abstract}
Background/Aim: Decreasing the vascularity of a tumour has proven to be an effective strategy to suppress tumour growth and metastasis. Anti-angiogenic therapies have revolutionized the treatment of advanced-stage cancers, however there is still demand for further improvement. This necessitates new experimental models that will allow researchers to reliably study aspects of angiogenesis. The aim of this study was to demonstrate an in vivo technique in which the highly vascular and accessible chorioallantoic membrane (CAM) of the chick embryo is used to study tumour-induced changes in the macro and microvessels. Materials and Methods: Two cancer cell lines (human melanoma (C8161) and human prostate cancer (PC3)) were selected as model cells. Human dermal fibroblasts were used as a control. One million cells were labelled with green fluorescent protein and implanted on the CAM of the chick embryo at embryonic development day (EDD) 7 and angiogenesis was evaluated at EDDs 10, 12 and 14. A fluorescently-tagged lectin (lens culinaris agglutinin (LCA)) was injected intravenously into the chick embryo to label endothelial cells. The LCA is known to label the luminal surface of endothelial cells, or dextrans, in the CAM vasculature. Macrovessels were imaged by a hand-held digital microscope and images were processed for quantification. Microvessels were evaluated by confocal microscopy. Tumour invasion was assessed by histological
\end{abstract}

This article is freely accessible online.

Correspondence to: Prof. Sheila MacNeil, Tissue Engineering Group, Kroto Research Institute, University of Sheffield, North Campus, Broad Lane, Sheffield, S3 7HQ, U.K. Tel: +44 0114222 5995, e-mail: s.macneil@sheffield.ac.uk

Key Words: Angiogenesis, tumour intravasation, tumour thrombus, prostate cancer, melanoma. and optical sectioning. Results: Tumour cells (C8161 and PC3) produced quantifiable increases in the total area covered by blood vessels, compared to fibroblasts when assessed by digital microscopy. Tumour invasion could be demonstrated by both histological and optical sectioning. The most significant changes in tumour vasculature observed were in the microvascular structures adjacent to the tumour cells, which showed an increase in the endothelial cell coverage. Additionally, tumour intravasation and tumour thrombus formation could be detected in the areas adjacent to tumour cells. The fragility of tumour blood vessels could be demonstrated when tumour cells seeded on a synthetic scaffold were grown on CAM. Conclusion: We report on a modification to a well-studied CAM in vivo assay, which can be effectively used to study tumour induced changes in macro and microvasculature.

In 20128.2 million people worldwide died from cancer (1), mainly due to a lack of complete understanding of the biological processes leading to cancer metastasis. Tumour growth and metastasis are dependent on angiogenesis (2). The finding that blocking angiogenic pathways stops the progression of cancer represents a milestone in angiogenesis research (3). Anti-angiogenic therapies such as the antiVEGF antibody (Bevacuzimab) and VEGF receptor kinase inhibitors (sunitinib and sorafenib) are now indicated in the treatment of advanced stage cancers such as colon, lung, breast and kidney $(4,5)$. However, many patients do not show a sustained response to these agents (6). Resistance to therapy is common and it cannot currently be predicted (7). Additionally, some patients have less benefit from the treatment, indicating that different cancers vascularize with different mechanisms (8). Thus, targeting angiogenic pathways may be an effective strategy to fight against cancer however a better understanding of tumour vascularization and mechanisms leading to development of resistance is essential when developing new treatment agents (9). This 
can only be achieved by using relevant experimental models to study tumour angiogenesis.

Traditional methods to study tumour angiogenesis rely mainly on $2 \mathrm{D}$ cell cultures and small animal models. The obvious limitation of $2 \mathrm{D}$ culture methods is that they fail to recreate complex cell-cell and cell-matrix interactions in the tumour microenvironment (10). On the other hand, the available in vivo models are expensive, do not allow for high throughput screening and require expertize (e.g. dorsal skin fold assay) if visualization of blood vessels is required. Increasingly more research is focusing on constructing $3 \mathrm{D}$ in vitro models of tumour angiogenesis using tissue-engineering techniques $(11,12)$. However, this field is still developing.

The chorioallantoic membrane (CAM) assay stands out as an economical alternative bioassay to study tumour angiogenesis in vivo. The CAM provides researchers with a readily accessible dense network of visible arteries and veins that allows nutrients for efficient grafting of tumour cells. When a fluorescently tagged lectin (lens culinaris agglutinin (LCA)) is injected into the circulation of the chick embryo, the capillary plexus of the CAM can be visualized by a top planar view in the fluorescent microscope. The planar arrangement of this capillary plexus (microvasculature) and its proximity to the surface makes it a valuable model to study angiogenic processes at the cellular level, in both physiological and pathological situations. Additionally, the chick embryo is essentially immunodeficient in the initial stages of embryonic life, unlike murine models that do not support the growth of all human tumour types. More importantly, the CAM model offers distinctive advantages when studying angiogenesis.

Tumour angiogenesis is driven by several mechanisms including sprouting angiogenesis, intussusceptive angiogenesis and recruitment of endothelial progenitor cells, vessel cooption, vasculogenic mimicry and lymphangiogenesis (13). The first advantage of the CAM model is that it allows direct visualization of sprouting and intussusceptive angiogenesis, which can be of particular importance when alternative pathways in treatment resistance are, to be studied. Secondly, the CAM assay allows monitoring of tumour intravasation into the CAM microvasculature (14). Intravasation is entry of tumour cells into the vasculature and it is the most critical step in initiation of tumour metastasis (15). Intravasation is considered to be the rate-limiting step in cancer metastasis however it is under- investigated (16), due to the requirement for sophisticated technical imaging equipment (e.g. time-lapse confocal microscopy) that is required to study in vivo tumour intravasation and metastasis (17).

The aim of this study is to describe a methodology that allows evaluation and quantification of the effect of a very highly metastatic human melanoma cell line (C8161) and a prostate cancer cell line (PC3) in a CAM assay. We investigated the progressive growth of tumours grafted onto
CAM ectoderm, and established a reproducible and simple method to investigate capillary plexus (micro-vessels). We have also investigated a high-resolution imaging method to study intravasation in both wide diameter vessels (macrovessels in the mesoderm) and the capillary plexus (microvessels in the ectoderm) using this assay.

\section{Materials and Methods}

Culturing melanoma and prostate cancer cell lines and primary human fibroblasts. C8161 human cell line was isolated from an abdominal wall metastasis from a recurrent malignant melanoma of a menopausal woman (developed by Professor F. Meyskens UC Irvine (USA) via Dr. M. Edwards (University of Glasgow, UK)). C8161 human melanoma cells were grown in melanoma culture medium consisted of EMEM media (Sigma-Aldrich, Dorset, UK) supplemented with FCS $(10 \%$ v/v), L-glutamine $(2 \mu \mathrm{M})$, Pencillin $(100 \mathrm{U} / \mathrm{ml})$, streptomycin $(100 \mu \mathrm{g} / \mathrm{ml})$ and Fungizone $(0.625 \mu \mathrm{g} / \mathrm{ml})$.

The human prostate cancer cell line (PC-3) was a kind gift from Dr. Adam Glen (initially purchased from the American Type Culture Collection (Manassas, VA, USA)). PC-3 cells were cultured in T75 flasks and maintained in RPMI-1640 medium supplemented with FCS $(10 \% \mathrm{v} / \mathrm{v})$, L-glutamine $(2 \mu \mathrm{M})$, Penicillin $(100 \mathrm{U} / \mathrm{ml})$, streptomycin $(100 \mu \mathrm{g} / \mathrm{ml})$ and Fungizone $(0.625 \mu \mathrm{g} / \mathrm{ml})$ (all from Sigma-Aldrich, Dorset, UK).

Human skin fibroblasts (HDF) were isolated from the dermal part of split-thickness skin grafts as described previously $(18,19)$. After isolation HDFs were expanded in DMEM medium (Sigma-Aldrich) supplemented with FCS $(10 \% \mathrm{v} / \mathrm{v})$, L-glutamine $(2 \mu \mathrm{M})$, penicillin $(100 \mathrm{U} / \mathrm{ml})$, streptomycin $(100 \mu \mathrm{g} / \mathrm{ml})$ and Fungizone $(0.625 \mu \mathrm{g} / \mathrm{ml})$ and used between passages 3 and 9. HDF isolation, storage and experimentation was carried under a Local Ethics Committee (Sheffield NHS Trust, Sheffield, UK) Approval and all tissue is banked under a Human Research Tissue Bank License Human Tissue Authority no 12179. All three cells types were initially grown until $80 \%$ confluent in $\mathrm{T} 75$ flask before detaching, counting, adjusting to required cell number and seeding.

\section{Studying angiogenesis}

Incubation of eggs. Pathogen-free fertilized white leghorn chicken eggs (Gallus gallus domesticus) were obtained from Henry Stewart Co. Ltd (UK). Care was consistent with the guidelines of the Home Office, UK. Chick embryos were cultured as described previously (20). The egg shells were cracked and embryos were transferred into a square Petri dish on embryonic development day (EDD) 3 . The $e x$ ovo cultures were maintained in a humidified incubator at $38^{\circ} \mathrm{C}$ between EDDs 3 to 14. The survival of the embryos were checked daily and recorded.

The ex ovo CAM assay. C8161, PC-3 and HDF cells were prelabelled with 1:1,000 concentration of Celltracker green ${ }^{\mathrm{TM}}$ (Invitrogen) and implanted on the CAM at embryonic development day (EDD) 7, at an initial seeding density of $1 \times 10^{5}$. Firstly, a plastic ring obtained by cutting a $2 \mathrm{~mm}$ thick slice of a $30 \mathrm{G}$ needle cover was placed on the CAM (Figure 1A). Then a microtrauma was applied on the CAM by gently touching with the bulb of a sterile Pasteur pipette. All chick embryos were then randomly allocated to either HDF, C8161 and PC- 3 groups (5 chick embryos in each group per experiment for 3 independent 
A

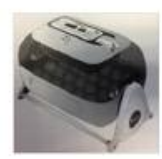

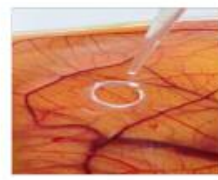

EDD 7
EDD 0 Incubation started

Cell implantation

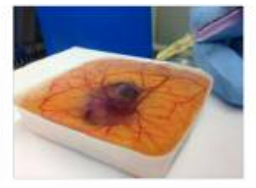

EDD 10, 12 \&14

Microinjection

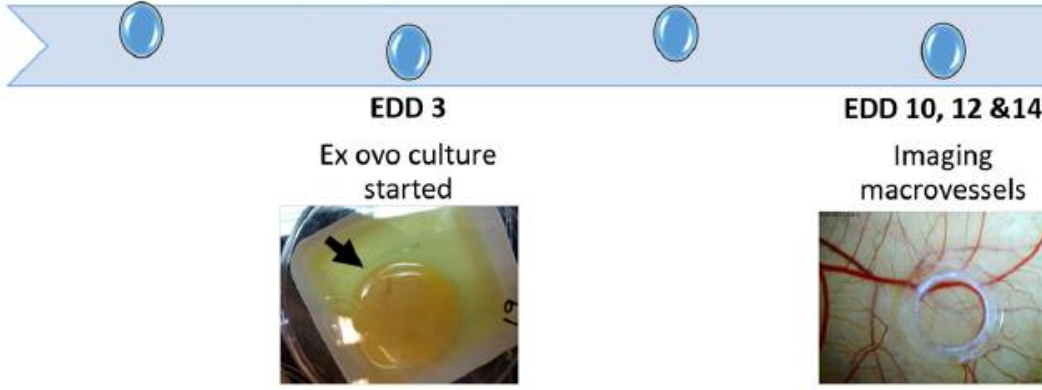

B
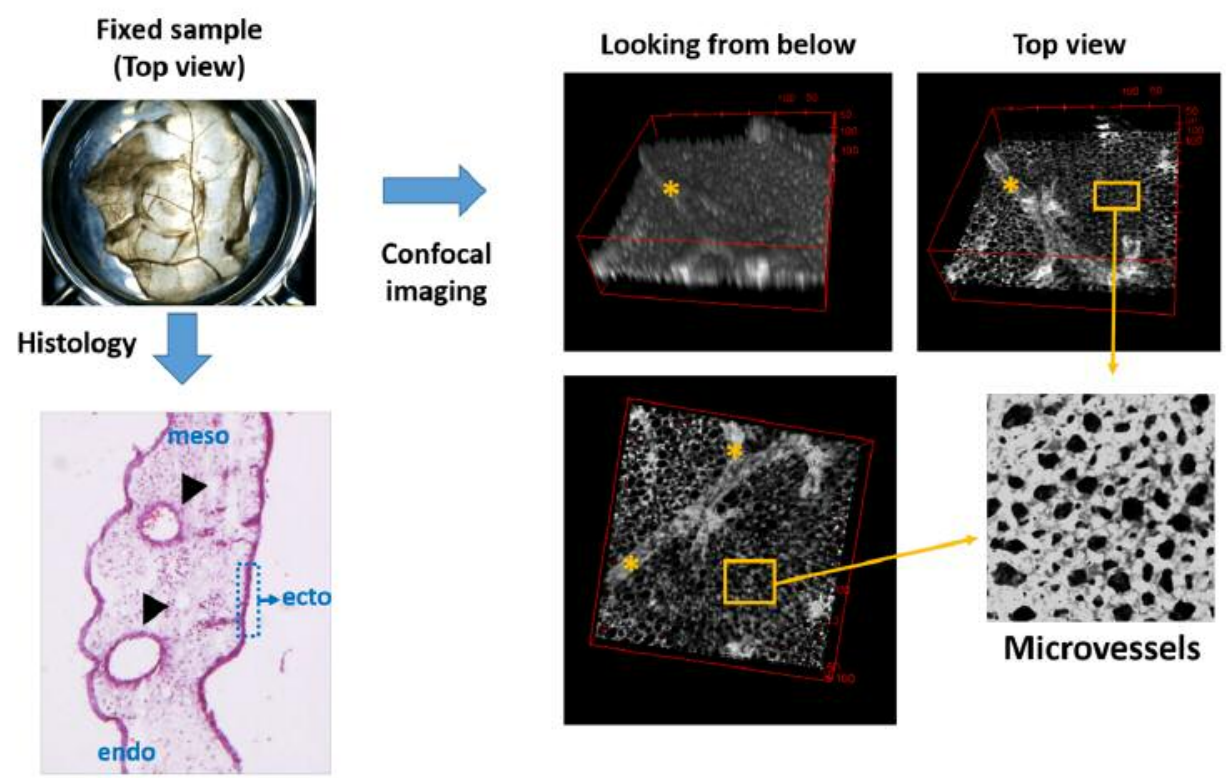

Cross sectional view

Figure 1. Schematic demonstration of running a CAM assay and the methodology used to image the macro and microvessels on the chorioallantoic membrane (CAM) of the chick embryo. (A) Flow of events from embryonic development day (EDD) 0 to EDD 14 during ex ovo culture of chick embryos. Incubation of fertilized eggs was started at EDD 0 and embryos were transferred into plastic weighing boats by cracking egg shells at EDD 3. Fluorescent labelled cells were implanted onto the CAM using a plastic ring at EDD 7. Experimental outcomes were evaluated at EDDs 10, 12 and 14 where macrovessels were first imaged by a hand-held microscope. Then microinjection of a fluorescent tagged lectin was performed and embryos were sacrificed. (B) Histological sectioning of the CAM reveals the cross-sectional view of the three-layered structure composed of ectoderm, mesoderm and endoderm from top to bottom. The macrovessels are located in the mesoderm (arrow heads) and the microvessels (capillary plexus) are located in the densely stained ectoderm. Confocal micrograph images of LCA-labelled endothelial cells of the vasculature shows both macro (asterisk) and microvessels in different depths of focus.

experiments). One million HDF, C8161 or PC3 cells suspended in $30 \mu \mathrm{l}$ of PBS were pipetted onto the CAM circumscribed with plastic ring at EDD 7. At EDD 10, 12 and 14, primary area and surrounding CAM images were taken by a digital microscope. For fluorescent angiography $50 \mu \mathrm{l}$ of a $5 \mu \mathrm{g} / \mathrm{ml}$ of rhodamine labelled lens culinaris agglutinin (LCA) (Vector laboratories) was injected in peripheral veins of the viable CAM using a $30 \mathrm{G}$ hypodermic needle attached to a $1 \mathrm{ml}$ syringe. After injection the needle was withdrawn, haemostasis was established using a cotton swab and the embryos were incubated for another 3 minutes to let the LCA circulate. Embryos were then sacrificed by cutting the vitelline arteries. CAM areas labelled with plastic rings were cut with a 1 $\mathrm{cm}$ margin around them and fixed in $3.7 \%$ paraformaldehyde (Sigma Aldrich, Dorset, UK) in PBS. 
The above method of seeding was compared in another experiment, where 1 million C8161 cells were seeded on electrospun poly(lactic acid) (PLA) scaffolds or encapsulated in a gelatin based UV crosslinked hydrogel (21) for 24 hours and implanted on CAM (EDD 7).

\section{Quantification of angiogenesis}

Macrovessels. HDFs, C8161 and PC-3 tumour cells were cultured on the CAM until EDD 14. Digital images of the area of implantation were taken at EDD 10,12 and 14 using a digital microscope. Quantification of angiogenesis was performed on 6 digital images from each group following the processing methodology described previously (22). Briefly, images were converted to grayscale, adaptive thresholding was performed, noise removed and the percentage of area covered by blood vessels was calculated.

Microvessels. The microvasculature was imaged by confocal microscopy after focusing on the capillary plexus located just above the larger blood vessels on the top view of the fixed CAM tissues (Figure 1B). Quantification of endothelial cell hypertrophy in relation to implantation of tumour cells or HDFs in the adjacent CAM tissues was studied on processed images constructed using Image J software. Original images were converted to grayscale, 'enhance contrast' applied, bandpass filter applied and images were 'thresholded' to be most representative of original images. The total area covered by endothelial cells in a frame was calculated. The frames were taken from randomly selected areas of the CAM adjacent to HDF or tumour cells.

Studying tumour invasion

Confocal imaging. Fixed CAM samples were imaged at 3 time points $(10,12$ and 14 days) to track the growth of tumour cells implanted on CAM ectoderm and intravasation of tumour cells in endothelial cells by confocal microscopy. A Zeiss LSM 510 META confocal upright microscope with Zeiss LSM Image Browser software (version 4.2.0121) was used to analyze the data. EC-plan Neofluar 5X and 10X objective lenses was used (Celltracker green was excited at $488 \mathrm{~nm}$ and emitted at BP500-550 nm, while rhodamine LCA was excited at $543 \mathrm{~nm}$ and emitted at BP565-615 nm). In depth analysis of tumour implanted on the CAM optical images were taken at every $25 \mu \mathrm{m}$ interval, confocal settings (frame size $512 \times 512$, scan direction (single), scan speed (4), data depth ( 8 bit), pinhole (1 Airy unit), laser power and detector gain were all kept constant for all samples. Tumour engraftment was defined as presence and proliferation of cells on CAM at EDD 10,12 and 14. Presence of cells was demonstrated by confocal imaging of green fluorescent labelled cells on CAM vasculature stained with LCA (CAM vessels through the sample, Figure 1C). Proliferation was determined by an increase in mass of cells at EDD 10, 12 and 14. Tumour invasion and intravasion was also noticed consistently when the full depth of samples was imaged using confocal z-stack settings.

Histology. Tissues were fixed in $3.7 \%$ paraformaldehyde (Sigma Aldrich, USA) in PBS for at least $1 \mathrm{~h}$. Histology was performed on the retrieved samples. They were placed into molds for cryosectioning filled with OCT solution (Leica, Germany). Samples were left to freeze at $-80^{\circ} \mathrm{C}$ and $10 \mu \mathrm{m}$ sections were cut with a cryostat (Leica CM1860UV, Leica Germany). Slides were then stained with haematoxylin \& eosin (H\&E), according to the standard protocol for frozen slides. Slides were then covered with
Table I. Percentage area covered by blood vessels (quantification of processed images in Figure 3B).

\begin{tabular}{lccc}
\hline & $\begin{array}{c}\text { Day 10 } \\
\%( \pm \text { SD })\end{array}$ & $\begin{array}{c}\text { Day 12 } \\
\%( \pm \text { SD })\end{array}$ & $\begin{array}{c}\text { Day 14 } \\
\%( \pm \text { SD })\end{array}$ \\
\hline HDF & $16.02( \pm 4,95)$ & $20.21( \pm 5,27)$ & $25,30( \pm 5,69)^{+}$ \\
C8161 & $18.02( \pm 5.21)$ & $26.28( \pm 6.39)^{+}$ & $35.52( \pm 7.07)^{*},+$ \\
PC3 & $18.23( \pm 4.29)$ & $34.17( \pm 6.63)^{*},+$ & $38.50( \pm 7.67)^{* *},+$ \\
\hline
\end{tabular}

${ }^{*} p<0.05$ compared to HDF on the same day. ${ }^{* *} p<0.005$ compared to $\mathrm{HDF}$ on the same day. ${ }^{+} p<0.05$ compared to day 10 on the same group. $\mathrm{n}=5$ for each time point in each group.

DPX mountant (Sigma-Aldrich, Dorset, UK), covered with a glass coverslip and imaged using a light microscope (Motic, China). Tumour invasion was defined as presence of cells in the mesoderm of the CAM as demonstrated by light and fluorescence microscopy imaging of histologic sections (Figure 2).

Statistical analysis. Statistical analysis was performed with SPSS v. 17.0. Differences between group means were analysed by Student's unpaired T-test when the data was normally distributed. Comparisons of more than 2 groups was performed with one-way ANOVA to determine the statistical significance. A $p$-value of $<0.05$ was considered statistically significant.

\section{Results}

Evaluation of effect of engrafted HDFs or tumour cells on macrovessels of the CAM. A progressive increase in blood vessels formation was noticed in all micrographic images. Visual inspection of images demonstrated an increase in blood vessel formation at EDD 14 versus EDD 10 in all cell lines (Figure 3A). It was also observed that there was a normal increase in formation of blood vessels in the developing embryo from EDD 10 to 14, though an enhanced angiogenesis was noticed in the tumour group compared to HDF group. The percentage areas covered by blood vessels at days 10, 12 and 14 in HDF, C8161 and PC3 groups are given in Table I. In all groups the total area covered by blood vessels increased from EDD 10 to EDD 14 however the response to C8161 and PC3 cells was statistically greater than that to HDFs. There was no difference between groups by EDD 10 but there was a significant difference between HDF and HDF and PC3 groups on EDD 12 and between C8161 and PC-3 cells and HDFs by EDD 14 (Figure 3).

Implantation of tumour cells on CAM using tissue scaffolds. When tumour cells (C8161) were seeded on polylactic acid (PLA) scaffolds and implanted on the CAM, a visible bleeding area inside the scaffold-tumour complex could be observed in 9 out of 18 implanted samples $(50 \%)$ as compared to no bleeding in the 12 of the control PLA scaffolds (Figure 3C). When tumour cells were encapsulated 


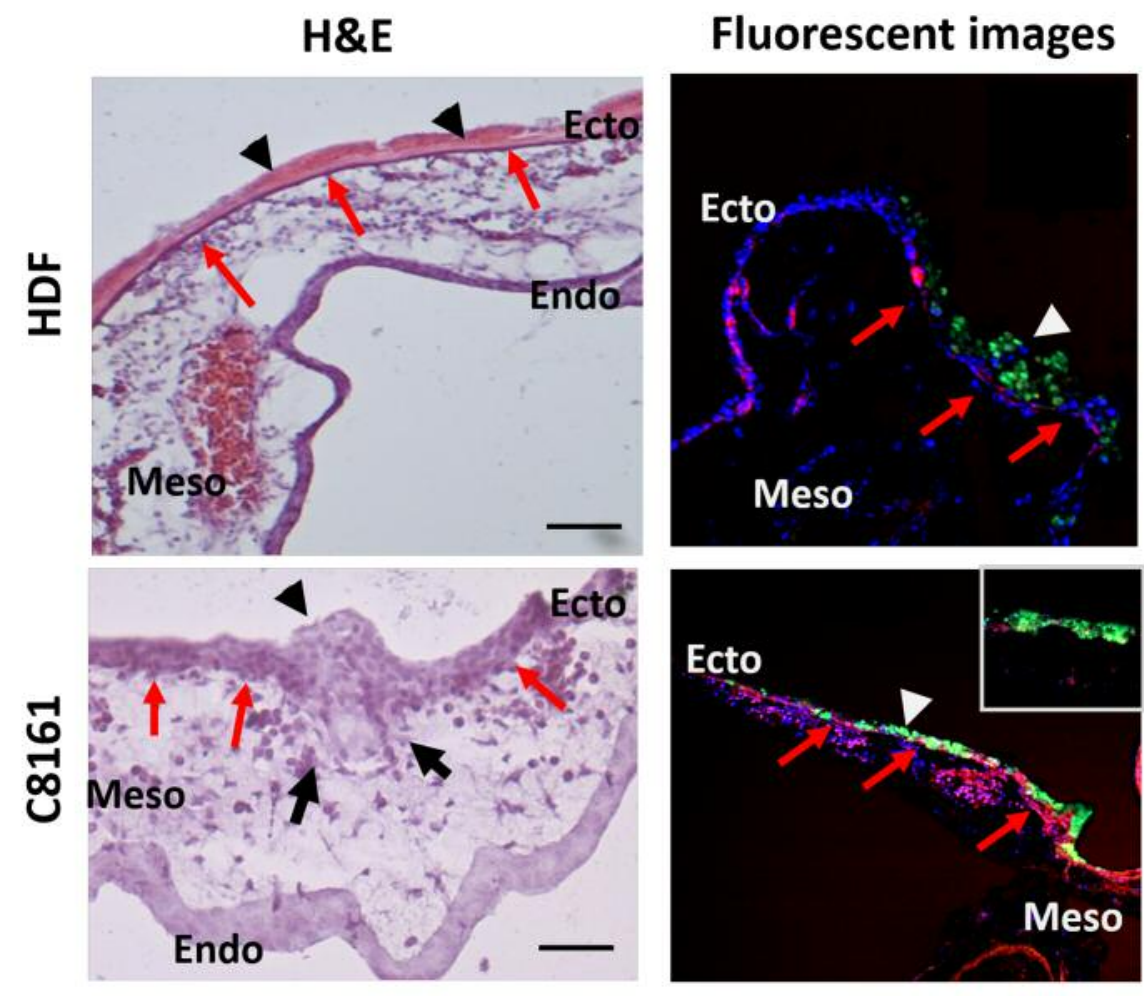

Figure 2. Methodology used to demonstrate tumour invasion by histological sectioning (haematoxylin and eosin (H\&E) and fluorescent labelled images). Upper row: Human dermal fibroblasts (HDFs) are seen on top of the ectoderm together with the extracellular matrix (ECM) (arrow heads) they have produced. The ectoderm of the CAM (red arrows) can clearly be followed underneath the HDF and ECM layer demonstrating noninvasion. Corresponding fluorescent micrographs confirm the presence of green fluorescent labelled HDFs on top of the ectoderm (red arrows), with no presence of HDFs in the mesoderm. Lower row: Human melanoma cells (C8161) can be seen as an aggregate (arrow head) on top of CAM extending from the surface to the mesoderm in $H \& E$ sections. The ectoderm-mesoderm border (red arrows) is destructed (black arrows). Corresponding fluorescent images confirm presence of fluorescently labelled tumour cells in the mesoderm of the CAM (green labelling/white arrow head) with disruption of the ectoderm-mesoderm border (red arrows) confirming invasion (see inset).

in the hydrogel before implantation on the CAM, no visible bleeding could be observed in all of the 14 samples, most probably due to poor tissue integration of the hydrogel and the CAM. Instead the hydrogel carrier system appeared to be good at demonstrating the tortuosity of the blood vessels adjacent to hydrogel-tumour complex. After these initial experiments we decided to implant the tumour cells directly on CAM without using a cell carrier, as different matrices demonstrated different responses in tumour vasculature.

Evaluation of primary tumour implantation site on CAM. The HDFs and tumour cells on the primary inoculation site demonstrated progressive proliferation at EDD 10, 12 and 14. Tumour / HDF cells were pre-labelled with cell tracker green while the endothelial cells were labelled with LCA. A progressive growth of all 3-cell types was observed. Interestingly, C8161 melanoma cell lines were found in a large cellular mass compared to HDF and PC3 prostate cells (Figure 4A, B). Individual tumour cells that detached from the primary tumour mass could also be observed intravasating from the lacuna to the cellular space (Figure 4C). Occasionally tumour thrombi could be detected inside relatively larger vessels (Figure 4C).

Demonstration of tumour invasion. Histologic sections of the primary site by inoculation demonstrated that all 3-cell types grew and formed cell masses or colonies on the ectoderm surface of the CAM by EDD 14. It was observed that HDFs formed a cellular uniform layer on top of the ectoderm that could be clearly differentiated (Figure 5A). The two distinct layers (HDF cell layer and ectoderm of CAM) confirmed non-invasiveness of HDF cells. It was also likely that the HDF uniform cellular layer was due to the formation of extracellular matrix produced at EDD 14 (Figure 5A). In contrast, C8161 and PC-3 cells formed cell mass/clumps unlike the HDF uniform ECM layer. The tumour cell mass appeared to have invaded the CAM ectoderm and even extended into the mesoderm (Figure 
A
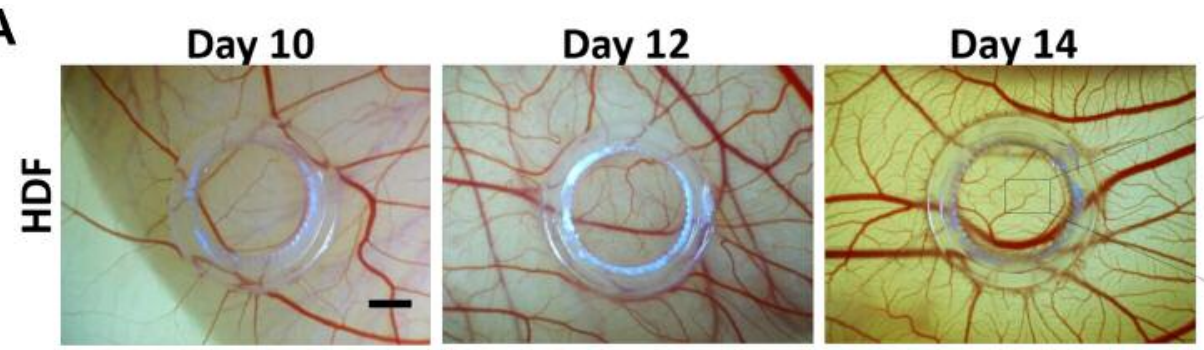

B
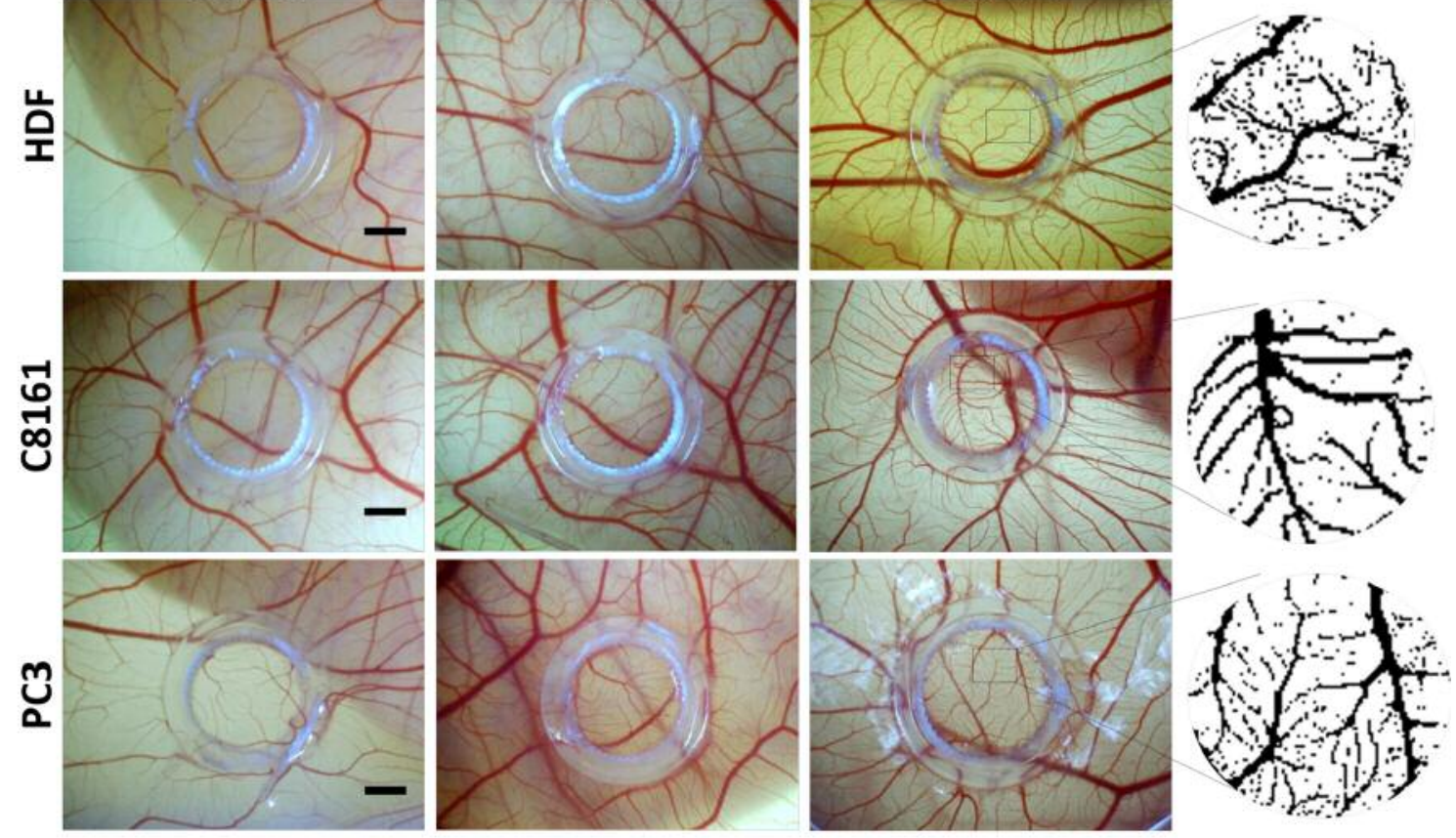

C

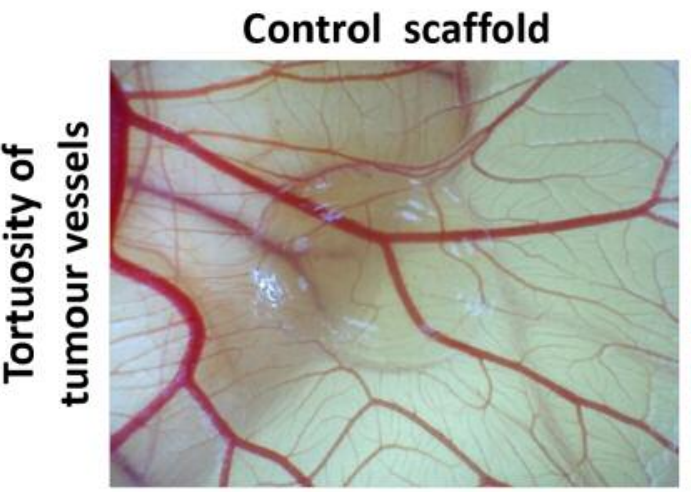

Scaffold with C8161
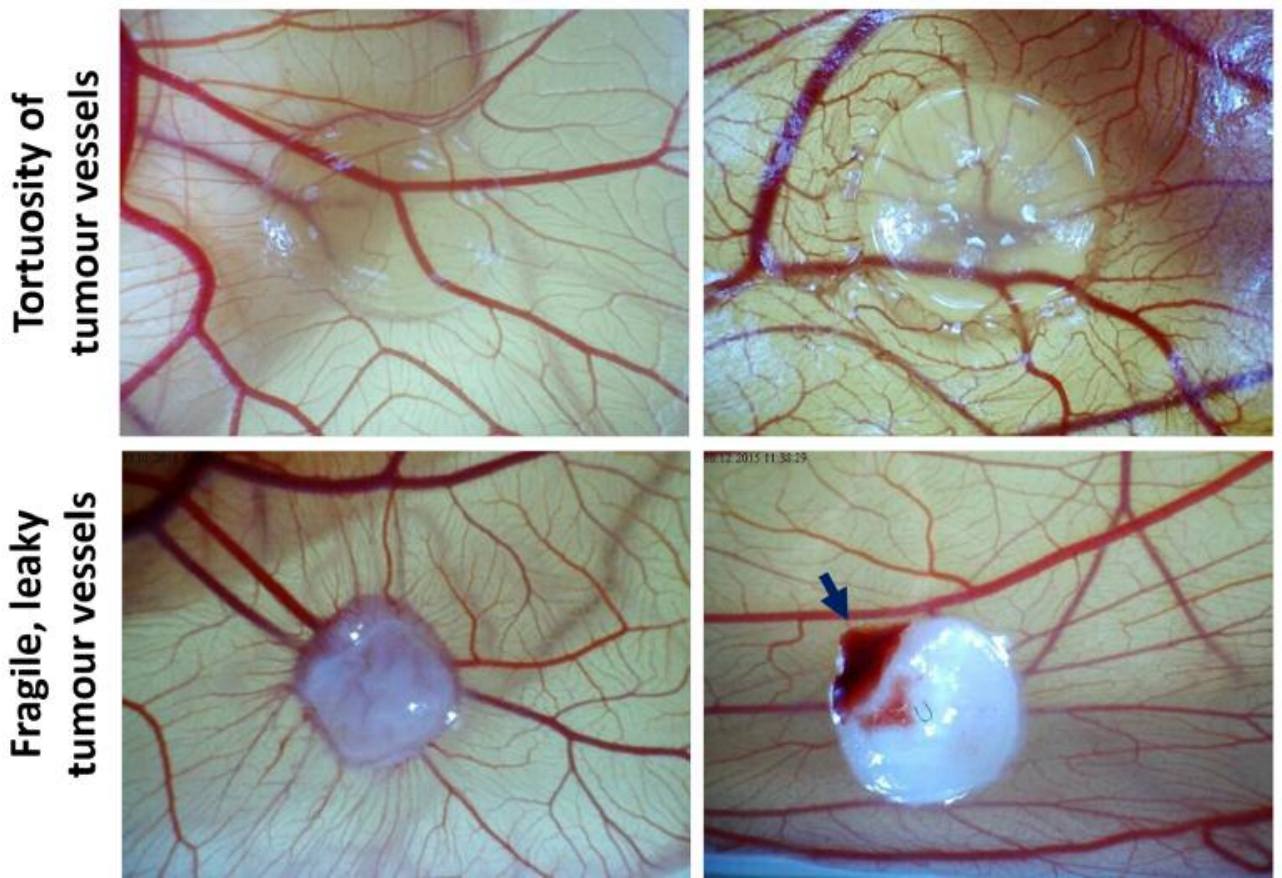

Figure 3. The gross appearance of tumour-induced angiogenesis on the area of implantation of the tumour cells/ fibroblasts on the chick chorioallantoic membrane (CAM). A) Micrographs of CAM regions at embryonic development days 10, 12 and 14 where human dermal fibroblasts (HDF) (upper row), melanoma (C8161) cells (middle row) and prostate cancer cells (PC-3) (lower row) were implanted. B) Processed images for each group at day 14 (quantification given in Table I). C) The quantification of images at B, the percentage area covered by blood vessels in each group at day 14. Scale bars represent $2 \mathrm{~mm}$. C) The tortuosity of tumour blood vessels can be observed when tumour cells are encapsulated in a transparent hydrogel and implanted on CAM for 7 days. The fragility of blood vessels can be demonstrated as spontaneous bleeding (with movements of the chick embryo) when tumour cells seeded on an electrospun PLA scaffold were cultured on CAM for 14 days. 
A
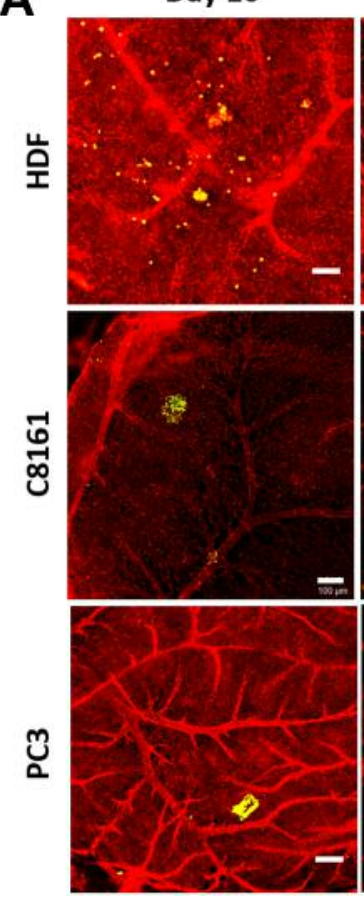

Day 12
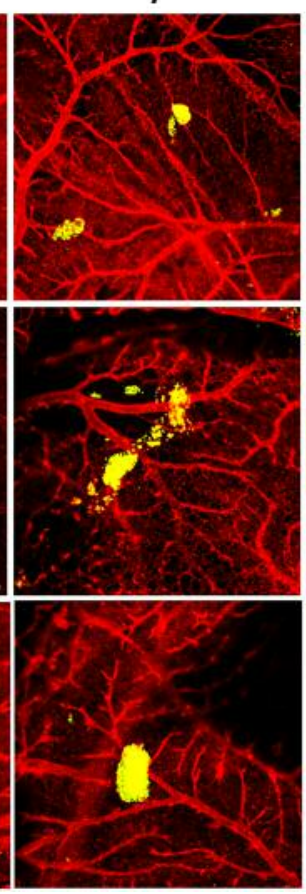

Day 14

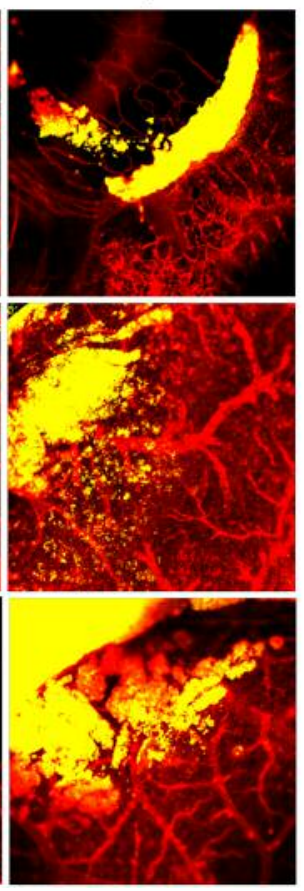

B
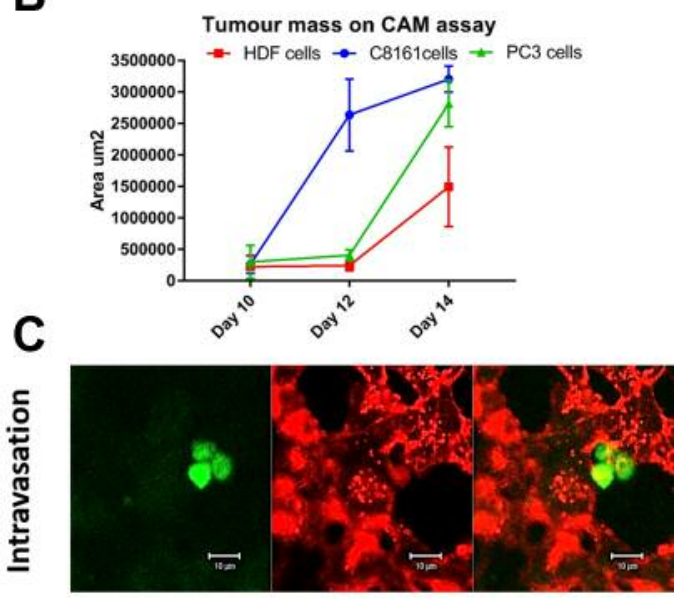

D

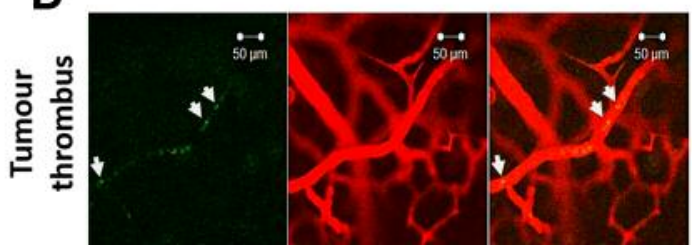

Figure 4. Confocal microscopic imaging of tumour-induced angiogenesis on the area of implantation of the cells on the chick chorioallantoic membrane (CAM). (A) Implanted human dermal fibroblasts (HDF), melanoma cell line (C8161) and prostate cancer cell line (PC-3) can be tracked on days 10,12 and 14 and the increase in tumour volume can be demonstrated to increase at the primary site of implantation over days. Scale bars represent $100 \mu \mathrm{m} .(B)$ Total increase in the main cell mass was most prominent in C8161, PC 3 and HDF respectively. (C) In closer view, individual cells that detaching from the main tumour mass could be detected intravasating into the endothelial cells in the adjacent areas. (D) Another tumour characteristic, tumour thrombi, can also be observed in the lager blood vessels. HDF, C8161 and PC3 cells are labelled with cell tracker green, endothelial cells are labelled with rhodamine conjugated lens culinaris agglutinin.

5A, B). Histology sections of CAM samples labelled with LCA for endothelial cells and cell tracker green for tumour cells were imaged by fluorescent microscopy (Figure 5B). The results showed similar clumps of cancer cells (C8161 and PC3) and a hypertrophied ectoderm. Furthermore, cellular invasion of tumour cells into ectoderm and mesoderm was also evident but it was not evident in HDF samples (Figure 5B). Both tumour cell lines (C8161 and PC3) demonstrated a consistent invasion in all samples, though the distance of invasion in ectoderm or mesoderm on EDDs 10, 12 and 14.

Tumour invasion was also studied using confocal z-stack imaging. $\mathrm{X}-\mathrm{Y}$ axis images were taken through the $\mathrm{Z}$-axis of samples every $25 \mathrm{um}$ (optical slice). The $\mathrm{z}$-stack image was then compressed to obtain an $\mathrm{X}-\mathrm{Y}$ projection (Figure 6, left), each emission depth was obtained using Depthcod (Ziess LSM Image Browser). Each image stack required images, including both microvessels (ectoderm) and macrovessels (mesoderm). This is because the method required optical sections to demarcate the two areas and it was difficult to clearly demarcate ectoderm and mesoderm when a large blood was not present at the same vertical position with the tumour. Our results suggest some areas of tumour mass (Figure 6 middle (green channel emission)) were at same depth as the macrovessels (Figure 6 right (green channel emission)), demonstrating the presence of tumour cells in the same plane as a large blood vessel of the mesoderm (Figure 6). This was evident for tumours, but not in case of HDF.

Evaluation of microvessels on the primary site of cell implantation. Figure 7A demonstrates the response of CAM microvasculature to known proangiogenic (VEGF) and antiangiogenic (sunitinib) drugs. Endothelial cell hypertrophy together with smaller lacunae can be observed in response to VEGF whereas endothelial cell coverage was significantly less with sunitinib treatment, which can be quantified (Figure 7B). A similar hypertrophy in the endothelial cells was observed in the microvasculature adjacent to C8161 and PC3 cells in contrast to HDF (Figure 7C). 
A

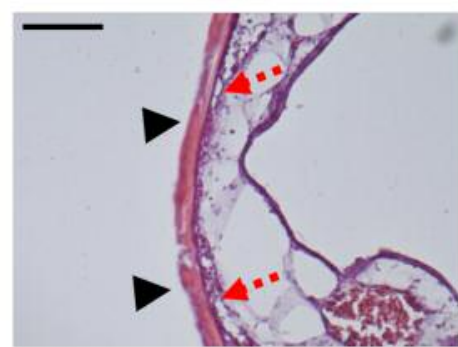

C8161

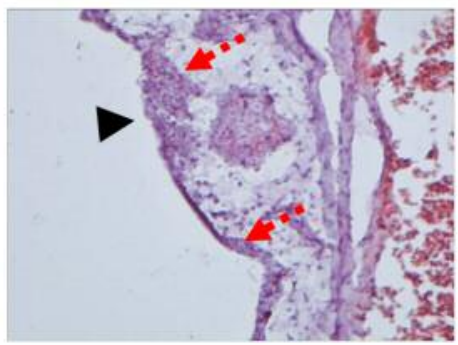

B

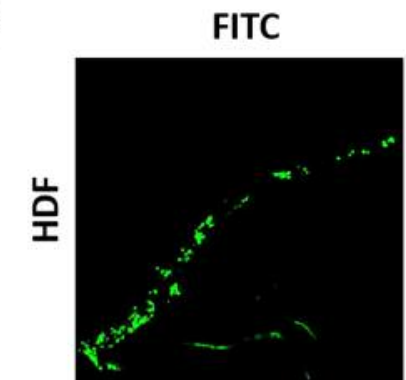

Rhodamine
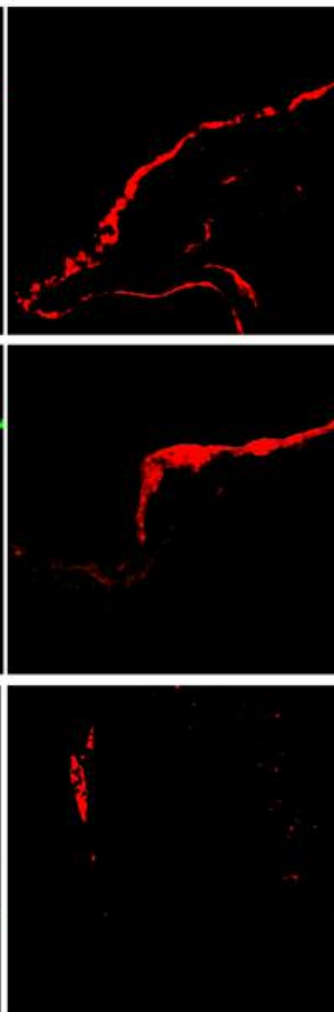

DAPI

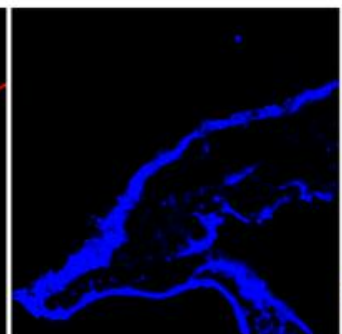

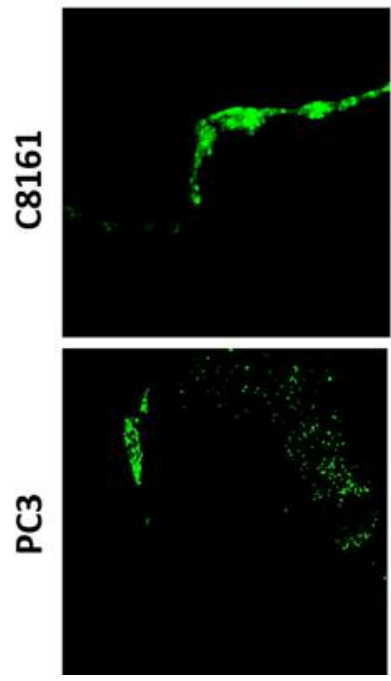
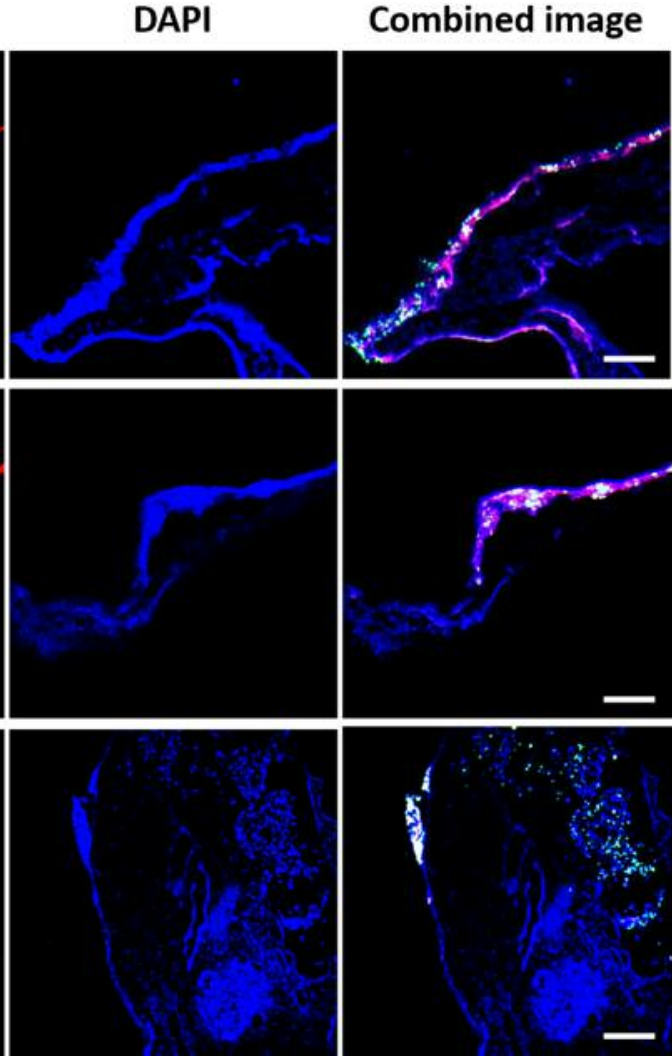

Figure 5. Evaluation of tumour invasion at the primary tumour site by histologic sectioning and fluorescent staining. A) $H \& E$ images demonstrate that fibroblasts $(H D F)$ grow on the surface without invading through the ectoderm of the CAM (dashed arrows) and a significant amount of ECM (arrow heads) can be observed on the CAM at day 14. With the melanoma (C8161) and prostate cancer (PC3) cell lines, tumour clumps can be seen on the surface of the CAM (arrow heads) and they damage the ectoderm extending through to the mesoderm (dashed arrows). B) Fluorescent staining demonstrates that green fluorescent labelled C8161 and PC3 cells invade through the ectoderm of the CAM. Invasion is defined as presence of tumour cells in mesoderm of the CAM.

\section{Discussion}

Accessible models to study tumour angiogenesis that are able to represent the complexity of $3 \mathrm{D}$ living systems are needed to study the mechanisms of tumour biology and progression as well as testing newly developed drugs. This study reports on an in vivo bioassay methodology based on the chick chorioallantoic membrane (CAM) that can be used for both qualitative and quantitative assessment of tumour angiogenesis at both macro and microscopic levels. We present a significant improvement on the classical assay by systematically combining several techniques to study some important aspects of tumour angiogenesis. We demonstrate that optical sectioning can be used in conjunction with histology sectioning to study tumour invasion, and that tissue engineered tumour constructs can be used to study the functional abnormality (fragile/leaky) of tumour vessels in an in vivo system. 

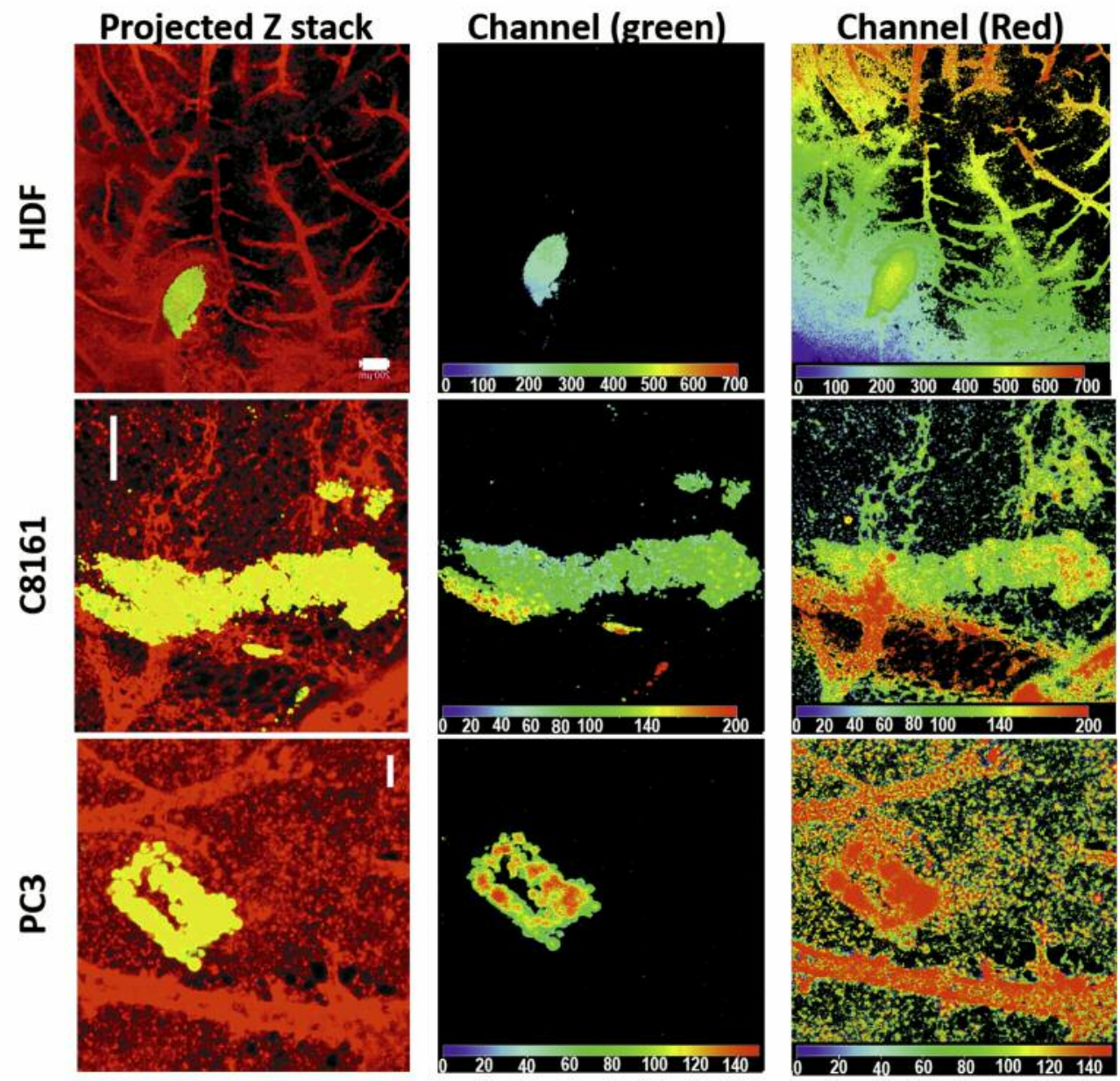

Figure 6. Demonstration of tumour invasion by optical sectioning in confocal microscopy. Projected Z stack images of all sections demonstrate HDF, C8161 and PC3 cells in relation to blood vessels of the CAM. Channel (green) locates the HDFs or tumour cells on the surface of and extending to the mesoderm of the CAM. Channel (red) locates the presence of blood vessels in the whole thickness of the imaged CAM. The depth map of the green and red channels taken together demonstrates extension of the tumour cells to the level of bigger blood vessels in the mesoderm confirming invasion. Scale bars represent $200 \mu \mathrm{m}$.

We report that all cells when placed on a highly vascularized chorioallantoic membrane of fertilised chick eggs grew over time. This approach provides a good alternative to the use of small animals, and offers an economical and fast experimental alternative to study tumour angiogenesis and invasion. Furthermore, we have demonstrated that 3D optical imaging of the CAM tissue can illustrate tumour growth and the tumour influence on angiogenesis. The CAM assay is a well-established assay that has long been used as an in vivo environment to study tumour metastasis and angiogenesis (23). Both in ovo (24) and ex ovo (shell less) (25) techniques have been used in previous studies to culture chick embryos. Although there is no major difference conceptually between the two methods (26), we selected the ex ovo technique as it allows better visualization of the blood vessels especially when studying angiogenesis and intravasation and it facilitates injections into the circulation. The main disadvantage of this technique is reported decreased embryo survival rates $(27,28)$. The survival rate in our hands for the ex ovo technique was above $70 \%$ (29).

We then demonstrated that the change in the overall appearance of the larger blood vessels can be quantified when tumour cells are implanted on a circumscribed area of CAM. While we explored several different methods of introducing melanoma cells onto the CAM (e.g. by placing cells within a hydrogel, or culturing them on a tissue engineered PLA scaffold), we found that the simplest approach was to use a simple cell suspension dropped within a light plastic ring placed on the CAM.

We used a published image analysis methodology to quantify the change in macrovessels (22). This methodology was applicable because we did not use a cell carrier material unlike previous studies that have used Matrigel or a collagen matrix to 


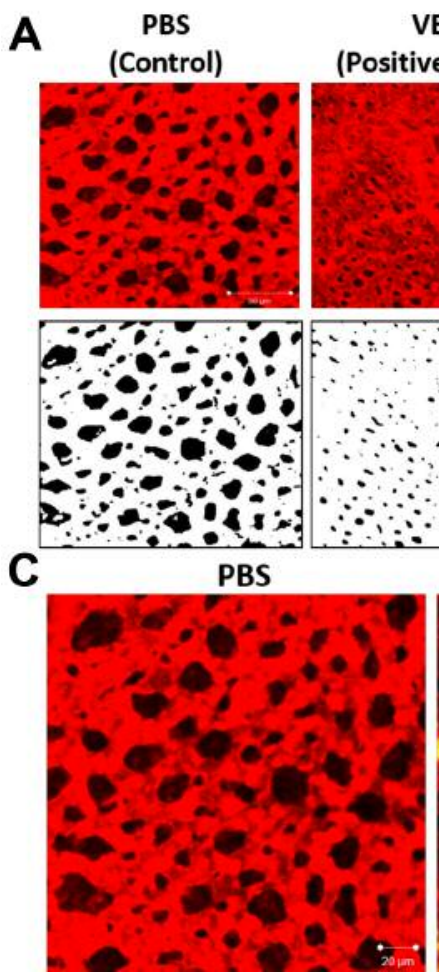

VEGF

Sunitinib
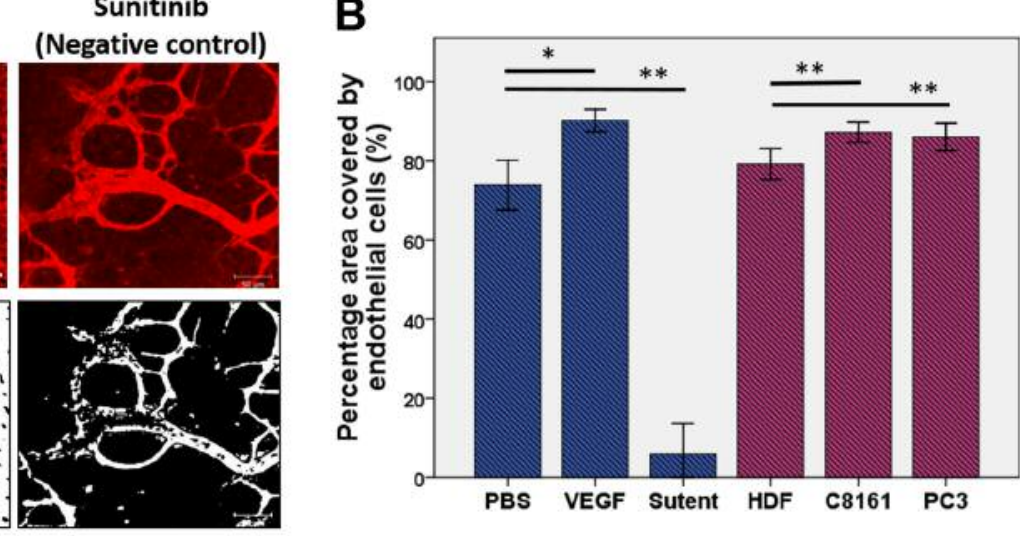

HDF

C8161
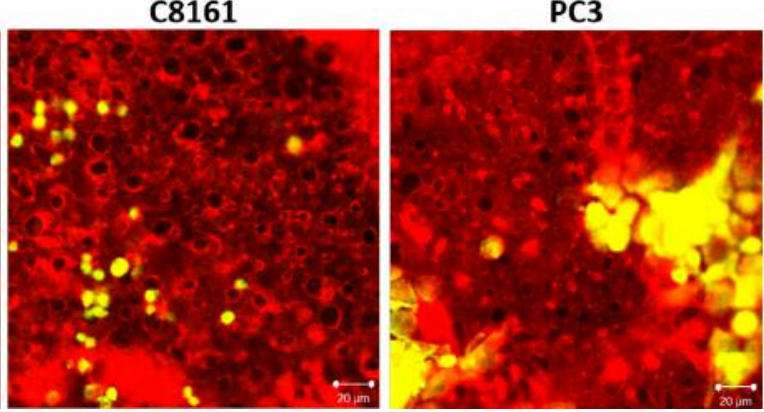

Figure 7. Demonstration of abnormal structure and function of tumour blood vessels. Evaluation of microcirculation adjacent to tumour cells. A) Demonstration of how CAM microvasculature appears normally (control) and how it responds to pro-angiogenic (VEGF) and anti-angiogenic (sunitinib) compounds. VEGF results in endothelial cell hypertrophy and smaller lacunea when applied to CAM surface whereas sunitinib results in much larger lacunae and areas of CAM devoid of endothelial cell coverage (avascular areas). B) Quantification of area covered by endothelial cells and the lacunea. C) In response to the presence of C8161 and PC3 cells, but not fibroblasts, local areas of endothelial cell hypertrophy can be observed in areas adjacent to tumour cells.

implant tumour cells on the CAM (17). Although using a cell carrier has obvious advantage of keeping tumour cells together, our initial experiments demonstrated a moderate inflammatory reaction to the natural ECM based carriers especially after EDD 12 (30). Inflammation is known to stimulate angiogenesis, which could also affect the quality of imaging.

The CAM, being a naturally immunodeficient host, is known to accept grafted tissues and cells (31-33). Furthermore, the CAM contains extracellular matrix proteins such as laminin, fibronectin, type I collagen, integrins and MMP-2 making it a useful model to study tumour invasion (34). Histological investigation in many studies have demonstrated invasion of the CAM ectoderm by tumours of different origins $(28,35)$. Here we present a demonstration of tumour invasion by optical sectioning, in addition to traditional histologic and fluorescent staining. By using confocal microscopy, we demonstrated the presence of tumour cells in the mesoderm of the CAM in cases where tumour inoculums were localized in close proximity to large blood vessels. One limitation of this technique was in the absence of adjacent large blood vessels and when the mesoderm was not thick enough, then the layers could not be differentiated by optical sectioning.
Following tumour growth, metastasis ensues by a cascade of events; detachment from the primary tumour, invasion of local stroma, entering the circulation (intravasation), surviving in the circulation, exiting from the circulation (extravasation) and engrafting in the metastatic site (36). With its accessible capillary plexus the CAM offers a unique environment to study tumour intravasation, dissemination and vascular arrest. Individual tumour cells that detach from the main mass intravasate to start the metastatic cascade. Tumour intravasation has also been studied in tumour masses formed in rats, however the visual data produced is rather unsatisfactory (37, 38) despite the specialized imaging techniques used.

In contrast to physiological angiogenesis, tumour related angiogenesis is characterized by structurally and functionally abnormal, disorganized, fragile and leaky vessels (39-41). An excessive production of VEGF together with a loss of balance between the pro and anti- angiogenic factors present in the tumour microenvironment mediates this process. We could demonstrate the tortuous blood vessels forming in relation to tumour cells by using a hydrogel as a cell carrier. This hydrogel is composed of cross linked gelatin (21) which makes it less antigenic and it does not integrate into CAM tissues allowing 
us to study the paracrine action of tumour cells. A stiffer extracellular matrix, electrospun PLA scaffold, was necessary to demonstrate the spontaneous bleeding as the chick embryo moved. To the best of our knowledge spontaneous bleeding inside the 3D tumour construct has not been demonstrated previously. One study demonstrated bleeding upon injection of tumour cells in a dorsal skin chamber (42).

A limitation of the current study is that only 2 established human cell lines were used to establish the model rather than excised human tumours. We chose human melanoma cells as this can spread locally and via the bloodstream (43). Also, it is known to be an angiogenic tumour type, whose aggressiveness is related to its vascularization status $(44,45)$. Prostate cancer cells were chosen as a solid tumour where antiangiogenic therapies have not shown any benefit despite the fact that angiogenesis plays a critical role in progression to metastatic prostate cancer (46). Tumours from different origins can be expected to induce different angiogenic responses.

Now that this model is established the next step will be to use it to study its angiogenic response to patient derived xenografts. We suggest this will be possible as previous studies have shown that solid tumours of different origins grown on CAM repeatedly formed solid tumours within days and their histologic appearances closely resembled the corresponding tumours from clinical specimens (47). Especially aggressive tumours for which treatments are largely ineffective (e.g. cholangiocarcinoma), tumour invasiveness and anti- tumour activity of new agents (e.g. metformin) can also be studied in this model (48). Metformin has also been suggested as a promising agent for metastatic prostate cancer when combined with the docetaxel chemotherapy (49) which is the current standard treatment (50). The challenge will be to see their response to anti-angiogenic drugs.

\section{Conclusion}

We report on a CAM assay as an effective methodology to study tumour-related changes in macro and micro blood vessels while allowing progressive tumour growth and invasion in an in vivo system. The main advantage of this approach is the fact that it offers an accessible approach to study tumour intravasation and we suggest that it will be amenable to use with human tumour biopsies and to determine the response of tumours to drugs in current clinical use. We hope it will become a useful model for providing much more information on the responsiveness of different tumours and of tumours from different patients to anti-angiogenic therapies.

\section{Acknowledgements}

The Authors thank the Rosetrees Trust for funding NM. The Authors also thank the Engineering and Physical Sciences Research Counsil (EPSRC) for funding AR.

\section{References}

1 Ferlay J, Soerjomataram I, Dikshit R, Eser S, Mathers C, Rebelo M, Parkin DM, Forman D and Bray F: Cancer incidence and mortality worldwide: sources, methods and major patterns in GLOBOCAN 2012. Int J Cancer 136: E359-386, 2015.

2 Hiratsuka S: Vasculogenensis, angiogenesis and special features of tumor blood vessels. Front Biosci 16: 1413-1427, 2010.

3 Bergers $G$ and Hanahan D: Modes of resistance to antiangiogenic therapy. Nat Rev Cancer 8: 592, 2008.

4 Jain RK: Antiangiogenesis strategies revisited: from starving tumors to alleviating hypoxia. Cancer Cell 26(5): 605-622, 2014.

5 Hurwitz H, Fehrenbacher L, Novotny W, Cartwright T, Hainsworth J, Heim W, Berlin J, Baron A, Griffing S and Holmgren E: Bevacizumab plus irinotecan, fluorouracil, and leucovorin for meta-static colorectal cancer. N Engl J Med 350(23): 2335-2342, 2004.

6 Simon T, Gagliano T and Giamas G: Direct effects of antiangiogenic therapies on tumor cells: VEGF signaling. Trends Mol Med 23(3): 282-292, 2017.

7 Jayson GC, Kerbel R, Ellis LM and Harris AL: Antiangiogenic therapy in oncology: current status and future directions. Lancet 388(10043): 518-529, 2016.

8 Döme B, Hendrix MJC, Paku S, Tóvári J and Tímár J: Alternative Vascularization Mechanisms in Cancer. Am J Pathol 170(1): 1-15, 2007.

9 Vasudev NS and Reynolds AR: Anti-angiogenic therapy for cancer: current progress, unresolved questions and future directions. Angiogenesis 17(3): 471-494, 2014.

10 Pampaloni F, Reynaud EG and Stelzer EH: The third dimension bridges the gap between cell culture and live tissue. Nat Reviews Mol Cell Biol 8(10): 839, 2007.

11 Bray LJ, Binner M, Holzheu A, Friedrichs J, Freudenberg U, Hutmacher DW and Werner C: Multi-parametric hydrogels support 3D in vitro bioengineered microenvironment models of tumour angiogenesis. Biomaterials 53: 609-620, 2015.

12 Zervantonakis IK, Hughes-Alford SK, Charest JL, Condeelis JS, Gertler FB and Kamm RD: Three-dimensional microfluidic model for tumor cell intravasation and endothelial barrier function. Proceedings of the National Academy of Sciences 109(34): 1351513520, 2012.

13 Hillen F and Griffioen AW: Tumour vascularization: sprouting angiogenesis and beyond. Cancer Metastasis Rev 26(3-4): 489-502, 2007.

14 Zijlstra A, Lewis J, Degryse B, Stuhlmann H and Quigley JP: The inhibition of tumor cell intravasation and subsequent metastasis via regulation of in vivo tumor cell motility by the tetraspanin CD151. Cancer Cell 13(3): 221-234, 2008.

15 Chiang SPH, Cabrera RM and Segall JE: Tumor cell intravasation. Am J Physiol Cell Physiol 311(1): C1-C14, 2016.

16 Deryugina EI, Zijlstra A, Partridge JJ, Kupriyanova TA, Madsen MA, Papagiannakopoulos T and Quigley JP: Unexpected effect of matrix metalloproteinase down-regulation on vascular intravasation and metastasis of human fibrosarcoma cells selected in vivo for high rates of dissemination. Cancer Res 65(23): 10959-10969, 2005.

17 Juncker-Jensen A, Deryugina EI, Rimann I, Zajac E, Kupriyanova TA, Engelholm LH and Quigley JP: Tumor MMP-1 activates endothelial PAR1 to facilitate vascular intravasation and metastatic dissemination. Cancer Res 73(14): 4196-4211, 2013. 
18 Ralston D, Laytont C, Dalley A, Boyce S, Freedlander E and MacNeil S: Keratinocytes contract human dermal extracellular matrix and reduce soluble fibronectin production by fibroblasts in a skin composite model. Brit J Plastic Surg 50(6): 408-415, 1997.

19 Ralston D, Layton C, Dalley A, Boyce S, Freedlander E and Neil SM: The requirement for basement membrane antigens in the production of human epidermal/dermal composites in vitro. Brit J Dermatol 140(4): 605-615, 1999.

20 De Magalhaes N, Liaw LH and Berns M: An instruction on the in vivo shell-less chorioallantoic membrane 3-dimensional tumor spheroid model. Cytotechnology 62(3): 279-283, 2010.

21 Eke G, Mangir N, Hasirci N, MacNeil S and Hasirci V: Development of a UV crosslinked biodegradable hydrogel containing adipose derived stem cells to promote vascularization for skin wounds and tissue engineering. Biomaterials 129: 188-198, 2017.

22 Doukas CN, Maglogiannis I and Chatziioannou AA: Computersupported angiogenesis quantification using image analysis and statistical averaging. IEEE Trans Inf Technol Biomed 12(5): 650657,2008

23 Deryugina EI and Quigley JP: Chick embryo chorioallantoic membrane model systems to study and visualize human tumor cell metastasis. Histochem Cell Biol 130(6): 1119-1130, 2008.

24 Palmer TD, Lewis J and Zijlstra A: Quantitative analysis of cancer metastasis using an avian embryo model. J Vis Exp 51: pii: 2815,2011

25 Subauste MC, Kupriyanova TA, Conn EM, Ardi VC, Quigley JP and Deryugina EI: Evaluation of metastatic and angiogenic potentials of human colon carcinoma cells in chick embryo model systems. Clin Exp Metastasis 26(8): 1033-1047, 2009.

26 Zijlstra A and Lewis JD: Visualization and quantification of de novo angiogenesis in ex ovo chicken embryos. The textbook of angiogenesis and lymphangiogenesis: Methods and applications. Springer Netherlands, 2012.

27 Cimpean AM, Ribatti D, Raica M: The chick embryo chorioallantoic membrane as a model to study tumor metastasis. Angiogenesis 11(4): 311-319, 2008.

28 Lokman NA, Elder AS, Ricciardelli C and Oehler MK: Chick chorioallantoic membrane (CAM) assay as an in vivo model to study the effect of newly identified molecules on ovarian cancer invasion and metastasis. Int J Mol Sci 13(8): 9959-9970, 2012.

29 Mangir N, Hillary CJ, Chapple CR and MacNeil S: Oestradiolreleasing biodegradable mesh stimulates collagen production and angiogenesis: An approach to improving biomaterial integration in pelvic floor repair. Eur Urol Focus 17, 2017. doi: 10.1016/j.euf.2017.05.004. [Epub ahead of print]

30 Roman S, Mangir N and Hearnden V: Angiogenic potential of adipose derived stem cells compared to the stromal vascular fraction. In: European Cells and Materials, AO Research Institute Davos pp. 40, 2016.

31 Lopez-Rivera E, Jayaraman P, Parikh F, Davies MA, Ekmekcioglu S, Izadmehr S, Milton DR, Chipuk JE, Grimm EA and Estrada Y: Inducible nitric oxide synthase drives mTOR pathway activation and proliferation of human melanoma by reversible nitrosylation of TSC2. Cancer Res 74(4): 1067-1078, 2014.

32 Balke M, Neumann A, Kersting C, Agelopoulos K, Gebert C, Gosheger $\mathrm{G}$, Buerger $\mathrm{H}$ and Hagedorn $\mathrm{M}$ : Morphologic characterization of osteosarcoma growth on the chick chorioallantoic membrane. BMC Res Notes 3: 58, 2010.

33 Dohle DS, Pasa SD, Gustmann S, Laub M, Wissler JH, Jennissen HP and Dunker N: Chick ex ovo culture and ex ovo CAM assay: how it really works. J Vis Exp 33: pii: 1620, 2009.
34 Giannopoulou E, Katsoris P, Hatziapostolou M, Kardamakis D, Kotsaki E, Polytarchou C, Parthymou A, Papaioannou S and Papadimitriou E: X-rays modulate extracellular matrix in vivo. Int J Cancer 94(5): 690-698, 2001.

35 Kunzi-Rapp K, Genze F, Kufer R, Reich E, Hautmann RE and Gschwend JE: Chorioallantoic membrane assay: vascularized 3dimensional cell culture system for human prostate cancer cells as an animal substitute model. J Urol 166(4): 1502-1507, 2001.

36 Yokota J: Tumor progression and metastasis. Carcinogenesis 21(3): 497-503, 2000.

37 Wyckoff JB, Jones JG, Condeelis JS and Segall JE: A critical step in metastasis: in vivo analysis of intravasation at the primary tumor. Cancer Res 60(9): 2504-2511, 2000.

38 Wang W, Wyckoff JB, Frohlich VC, Oleynikov Y, Hüttelmaier S, Zavadil J, Cermak L, Bottinger EP, Singer RH and White JG: Single cell behavior in metastatic primary mammary tumors correlated with gene expression patterns revealed by molecular profiling. Cancer Res 62(21): 6278-6288, 2002.

39 Fukumura D and Jain RK: Tumor microvasculature and microenvironment: targets for anti-angiogenesis and normalization. Microvasc Res 74(2-3): 72-84, 2007.

$40 \mathrm{McDonald}$ DM and Choyke PL: Imaging of angiogenesis: from microscope to clinic. Nat Med 9(6): 713-725, 2003.

41 Carmeliet P and Jain RK: Angiogenesis in cancer and other diseases. Nature 407(6801): 249-257, 2000.

42 Tozer GM, Akerman S, Cross NA, Barber PR, Bjorndahl MA, Greco O, Harris S, Hill SA, Honess DJ and Ireson CR: Blood vessel maturation and response to vascular-disrupting therapy in single vascular endothelial growth factor-A isoform-producing tumors. Cancer Res 68(7): 2301-2311, 2008.

43 Ribatti D, Annese T and Longo V: Angiogenesis and melanoma. Cancers (Basel) 2(1): 114-132, 2010.

44 Streit M and Detmar M: Angiogenesis, lymphangiogenesis, and melanoma metastasis. Oncogene 22(20): 3172-3179, 2003.

45 Bennicelli JL and Guerry Dt: Production of multiple cytokines by cultured human melanomas. Exp Dermatol 2(4): 186-190, 1993.

46 Mukherji D, Temraz S, Wehbe D and Shamseddine A: Angiogenesis and anti-angiogenic therapy in prostate cancer. Critical Rev Oncol/Hematol 87(2): 122-131, 2013.

47 Durupt F, Koppers-Lalic D, Balme B, Budel L, Terrier O, Lina B, Thomas L, Hoeben RC and Rosa-Calatrava M: The chicken chorioallantoic membrane tumor assay as model for qualitative testing of oncolytic adenoviruses. Cancer Gene Ther 19(1): 5868,2012 .

48 Saengboonmee C, Seubwai W, Cha'on U, Sawanyawisuth K, Wongkham S and Wongkham C: Metformin exerts anti-proliferative and anti-metastatic effects against cholangiocarcinoma cells by targeting STAT3 and NF-kB. Anticancer Res 37(1): 115-123, 2017.

49 Mayer MJ, Klotz LH and Venkateswaran V: Evaluating Metformin as a Potential Chemosensitizing Agent when Combined with Docetaxel Chemotherapy in Castration-resistant Prostate Cancer Cells. Anticancer Res 37(12): 6601-6607, 2017.

50 Mangir N and Türkeri L: Docetaxel based chemotherapy in the treatment of patients with castration resistant prostate cancer. Actas Urológicas Españolas (English Edition) 38(8): 515-522, 2014.

Received December 20, 2017

Revised February 7, 2018

Accepted February 8, 2018 